Article

\title{
Design and Position Control of Overhang-Type Rail Mover Using Dual BLAC Motor
}

\author{
Kiwan Cho and Dong-Hee Lee * \\ Department of Mechatronics Eng., Kyungsung University, 309, Suyeong-ro, Nam-gu, Busan 48434, Korea; \\ rldhks7201@ks.ac.kr \\ * Correspondence: leedh@ks.ac.kr
}

check for updates

Citation: Cho, K.; Lee, D.-H. Design and Position Control of Overhang-Type Rail Mover Using Dual BLAC Motor. Energies 2021, 14 , 1000. https://doi.org/10.3390/ en14041000

Received: 13 January 2021

Accepted: 8 February 2021

Published: 14 February 2021

Publisher's Note: MDPI stays neutral with regard to jurisdictional claims in published maps and institutional affiliations.

\begin{abstract}
This paper presents the design and position control scheme of an overhang-type rail mover system driven by a dual Brushless AC (BLAC) motor with a simple Hall position sensor inside each motor. The overhang-type roller is chosen to reduce the slip between the roller and rail surface due to gravity. The BLAC motors are used to provide smooth translation along the rail and effective turning. Imbalances on any part of the motor and the simplicity of the Hall position sensor can create additional disturbance load, unsteady movement, and position errors. To reduce the sudden moving position error between the two motors, a balancing compensator with a Proportional-Differential (PD) position controller, which is based on the instantaneous speed and position trajectories, is presented. Furthermore, speed and position reference models are designed to compensate for the low Hall sensor resolution in the low-speed range. Therefore, steady-state position errors can then be regulated simply by using the instantaneous speed and position information. Experiments were performed to verify the viability of the proposed system and control. The results show a significant improvement in roller translation along the rail and stopping position accuracy.
\end{abstract}

Keywords: rail traction system; BLACM; position control

\section{Introduction}

In the last decade, the demand for high-performance, unmanned surveillance equipment has increased due to the increase in unmanned facilities. One of the most popular systems is the rail mover. Rail movers are commonly used in industrial facilities, logistics, stage lightings, and unmanned security and monitoring systems [1-6]. In this paper, an overhang-type rail mover is proposed for autonomous monitoring systems. As the name suggests, the system consists of a moving body (mover) hanging on a guide rail with rollers on top, and it can transport equipment or has a camera attached for remote observation. Guide rails are commonly installed along the perimeter in a facility and usually are made of aluminum or iron with a slippery surface. The rollers are normally made from urethane, which has a strong adhesive property that creates friction, so that the mover can move along the rail without slipping. A supporting bar, however, is required for a mover that moves along a rail that is fixed to walls or ceilings. In this configuration, the attachment of the mover to the rail is a complicated process and can be costly. The supporting bar problem can be solved using a "hanging" roller instead. The structure looks like a swing hanging on a tree. However, the overhang configuration creates another problem: gravity-caused friction. Because of this, the friction between the rail and mover roller is inconsistent and varies during operation [7-9]. Some custom guide rails can be designed separately according to user needs [10-12]. These rails, though effective, are costly. Additionally, maintenance and repair are not easy because they are custom-made.

In a more advanced rail system, applications of a linear motor [13-15] and magnetic levitation $[16,17]$ can be found. As is well-known, a linear motor can guarantee high control performance with accurate position information; however, the moving rail has to contain permanent magnets or winding cores as a part of linear machines. Consequently, the 
system cost is greatly increased, and the installation is difficult. Furthermore, it requires an accurate linear position sensor. In the case of magnetic levitation systems, a specially designed guide rail, to suspend the mover body in the air, must be installed. Furthermore, it requires a complex control system with a levitation controller. For these reasons, linear motor and magnetic levitation systems are not proper for a low-cost, simple rail-guided moving systems. In general, a rotating motor attached to a roller to provide traction power is the most preferable choice in terms of cost and mechanical structure.

The overhang configuration is easy to implement; only some tension adjustments are required to compensate for the friction between the roller and rail because the tension tends to change according to mechanical vibrations and the downward force due to the weight and gravity of the mover. The proposed overhang-type mover, driven by a dual Brushless AC (BLAC) motor, has not been presented before. However, a similar overhead mover mounted on a crane with a pendulum-type load was researched [18-20]. Various observers and predictive control schemes have been adopted for the crane to reduce the mechanical vibration. The application considered in this study, however, is different than that of the crane. Unlike the pendulum-type load, the load here is strongly connected to the mover and roller. The main problem that needs to be solved through this research is, even though the motors are running together, the actual moving distance of each motor is not the same due to slip variations, friction, and different disturbance load [21]. Furthermore, an absolute position sensor cannot be installed on the rail to detect the actual translational moving length of the mover, which is normally estimated indirectly through rotation of the roller and traction motor. To maintain the tension in the conventional models, the equipment has to be adjusted frequently. Furthermore, a single roller type is more commonly used, but corner-turning is not easy to achieve. Gravity, which creates a downward force based on the weight of the system, can be used to decrease the slip effect, but the roller has to be installed on top of the rail instead of hanging below.

In this paper, an overhang-type rail mover system, using rollers which are driven with BLAC (Brushless AC) motors combined with a simple Hall position sensor, is presented. In a dual traction system, the actual moving speed and position of each motor is not the same. Because there are errors between each motor, the movement of the mover is unsteady and the instantaneous direction is not straight—it goes towards the left or right (zig-zag). The zig-zag movement may create collisions between the mover and rail, and this could lead to more unwanted friction. Furthermore, the mover also cannot precisely stop at the desired position. In this paper, the mechanical analysis of the proposed system is also presented to verify its structural safety.

A single inverter that can control both machines is preferred in a dual motor system to reduce cost and weight [22]; however, it cannot achieve precise position control. In the case of robots, backlash reduction can be implemented using a master-slave controller configuration [23]. However, this kind of motion controller does not deal with motor control, and a motor drive is usually equipped with an accurate encoder system. In the synchronization of linear motors, a complex quadratic performance index and crosscoupling control are presented [24], and the second-order sliding-mode control can be used as previously shown [25]. However, these presented controls are based on an accurate position sensor followed by complex mathematical models and pre-tested mechanical parameter validation.

A master controller with tri-cascade controls of dual BLDC (Brushless DC) motors is presented [26]. However, the position and speed feedbacks are considered as ideal signals, and the actual experiments were not performed. 
In the proposed system, two low-cost BLAC motors are utilized in the proposed rail mover system without using any absolute position sensors on the rail. To control the motor, a simple Hall position sensor is adopted, but the speed feedback delay and resolution have to be considered [9]. To improve the position control performance in the designed system, a time-based instantaneous speed and position reference control scheme is presented. In the proposed control, the moving position and speed are both controlled at the same time to precisely follow the desired speed trajectory. To satisfy the requirements of an overhang-type rail mover structure, a differential term, to control the instantaneous moving position, and a balance-compensating term are added. The differential control terms are based on the moving time of each position controller and can reduce sudden motion variations. The balancing error of the dual traction motor can also be reduced by the proposed compensation method.

The proposed method and system were tested and compared to the conventional control scheme. Through experiments, it can be confirmed that the proposed control scheme for the designed rail mover produces an advanced position control performance with steady motion.

\section{System Analysis}

\subsection{Mechanical Configuration}

Figure 1 shows detailed configurations of a conventional rail mover [9]. It is equipped with one HD (High Definition) camera for security and surveillance purposes. The camera motion is driven by a PMDCM (Permanent Magnet DC Motor), and it can move up and down as shown in Figure 1. Moreover, a BLAC motor is used for the mover to move forward and backward (FOR/REV).

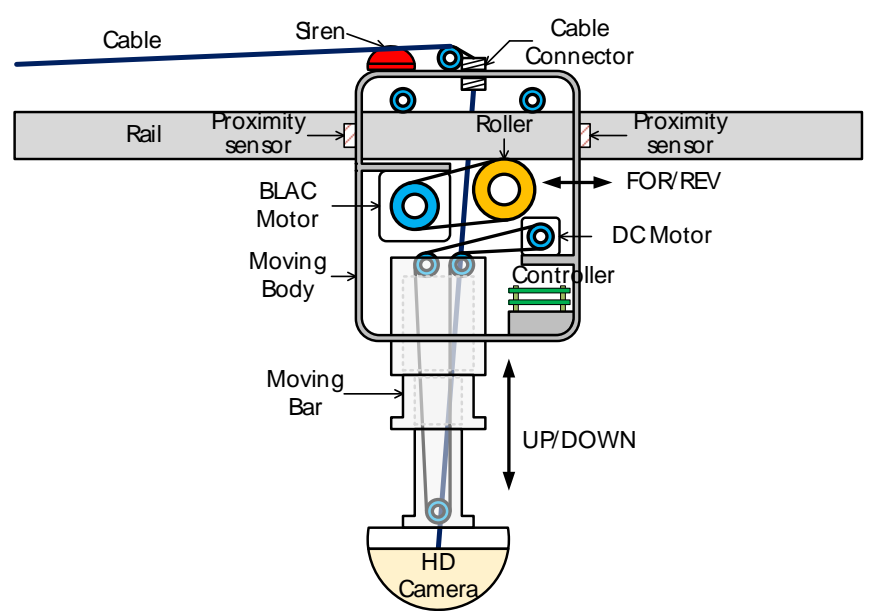

(a)



(b)

Figure 1. Conventional rail mover system: (a) overall structure; (b) mover traction with Brushless AC (BLAC) motor.

In a conventional rail mover, the guide rail is installed close to the ceiling, which means the roller is located at the bottom of the rail. Unlike the camera, the roller is rotating with BLAC motors using a belt and pulley. Gravity plays a big role in this configuration. Because of the weight of the mover system and gravity, the contact friction between the roller and rail surface changes accordingly to the moving velocity and rail condition. In Figure 2, the slip $s$ is caused by the lack of friction and friction variation between traction roller and rail surface. 


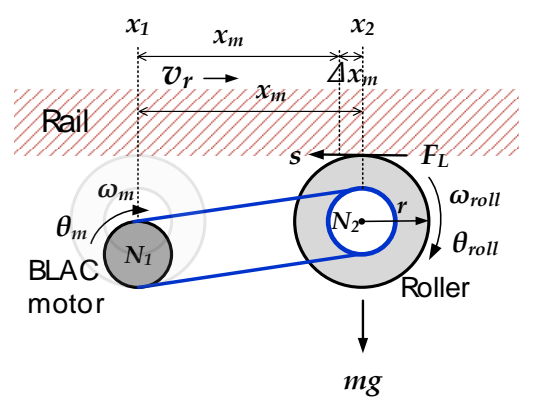

Figure 2. Mathematical model of roller (conventional structure).

Figure 2 shows the mathematical analysis of the conventional structure (roller below the rail). The mover velocity and moving length are expressed as $v_{r}$ and $x_{m}$. The load force and slip effect are $F_{L}$ and $s$. The rotating speed and moving angle of the roller and motor are noted as $\omega_{\text {roll }}, \theta_{\text {roll }}, \omega_{m}$ and $\theta_{m}$, respectively, with the subscript $m$ indicating the motor. The moving position of the mover (how much the mover had translated along the rail) can be described as follows:

$$
x_{2}=x_{1}+\int v_{r} \cdot d t
$$

Ideally, the motion velocity can be easily determined using the roller radius and rotating speed. However, because of the slip $s$ that occurs between the roller and the rail surface, the actual motion velocity $v_{r}{ }^{\prime}$ can be described as

$$
v_{r}{ }^{\prime}=r \cdot \omega_{m 1}{ }^{\prime}=r \cdot \omega_{\text {roll }} \cdot(1-s)
$$

Because of this slip, the actual moving position cannot exactly reach $x_{2}$ even though in the calculation it can because position error $\Delta x_{m}$ occurs. Moreover, the slip effect $s$ is not constant, and mechanical vibration makes the actual position more unpredictable. In order to reduce the slip effect, the downward force due to gravity has to be compensated.

Figure 3 shows the mechanical configurations of the proposed overhang-type rail mover using two separate geared BLAC motors. The motor is installed inside of the outer roller. Furthermore, the dual roller structure is detachable for easy installation. Guide rollers are placed on the side and below to avoid mechanical collision.

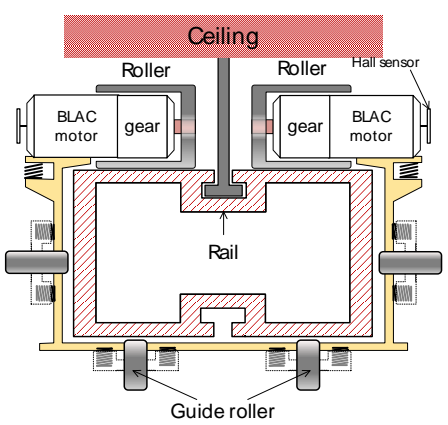

Figure 3. Proposed overhang-type rail mover using dual motors.

Figure 4 shows the mathematical model of the proposed overhang-type mover system. Gravity affects the roller and rail surface and increases the adhesivity with reduced slip during the translation of the mover on the rail surface. 


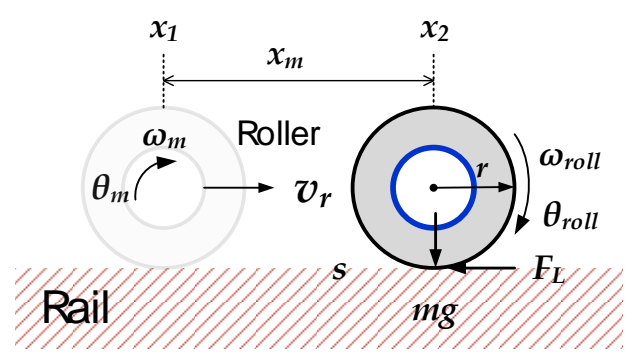

Figure 4. Mathematical model of the proposed system.

\subsection{Mechanical Configuration}

It can be seen that the overhang-type rail mover can increase the friction and reduce the roller-rail slip. However, it is difficult to make sure that both BLAC motors are rotating in the exact same way for smooth mover translation.

Figure 5 shows the moving characteristics of the proposed overhang-type rail mover driven by dual BLAC motors.

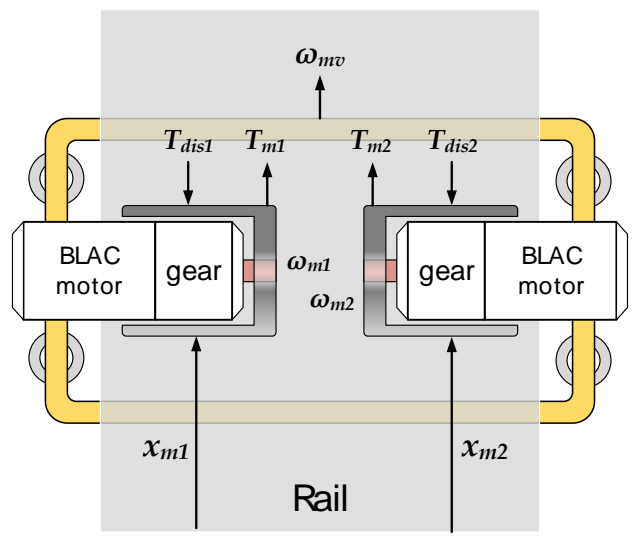

(a)

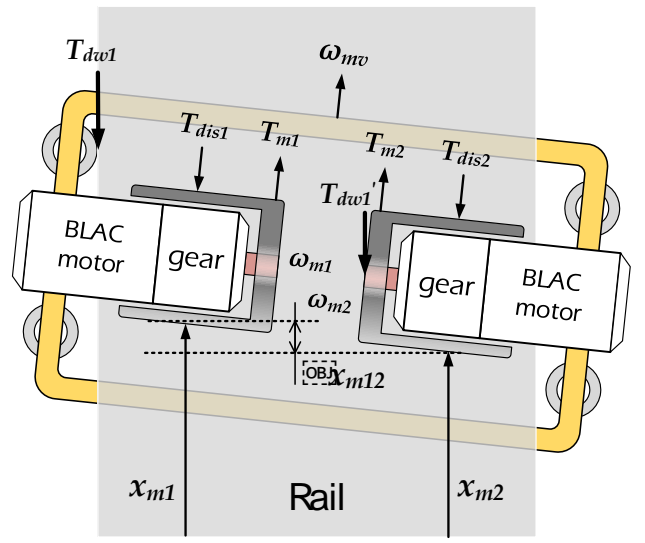

(b)

Figure 5. Moving characteristics in the overhang-type system: (a) ideal dual motor driving at the same speed; (b) actual moving from the velocity difference of two motors.

Ideally, as shown in Figure 5a, the moving length $x_{m 1}$ and $x_{m 2}$ will be the same if they have the exact same velocity and slip condition.

\section{Analysis of Overhang-Type Rail Mover Using Dual BLAC Motors}

\subsection{Mechanical Analysis of the Proposed Structure}

For stable operation and easy assembly, the mechanical structure of the proposed system is first analyzed using FEM (Finite Element Method).

The mechanical analysis results are shown in Figure 6. The weight of the body is assumed to be $10 \mathrm{~kg}$. The mechanical stress and displacement of the connecting part of the roller and motor frame are analyzed by ANSYS tools. The maximum mechanical stress is $16 \mathrm{MPa}$ at the fixation ring between the motor and mover body. The maximum mechanical displacement is $0.25 \mu \mathrm{m}$, which is caused by stress and weight at the same fixation ring shown in Figure $6 \mathrm{~b}$,c. However, aluminum material can yield up to $280 \mathrm{MPa}$, so the designed overhang-type rail mover can be considered mechanically stable. 


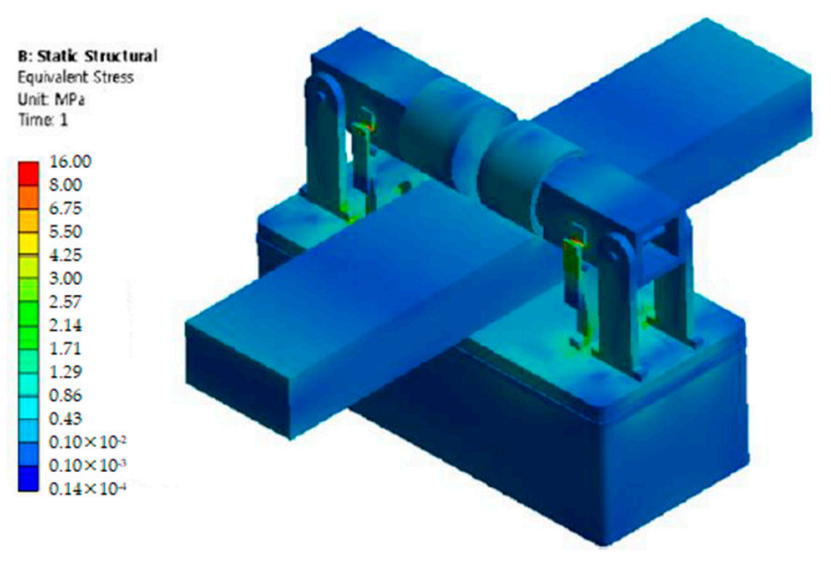

(a)

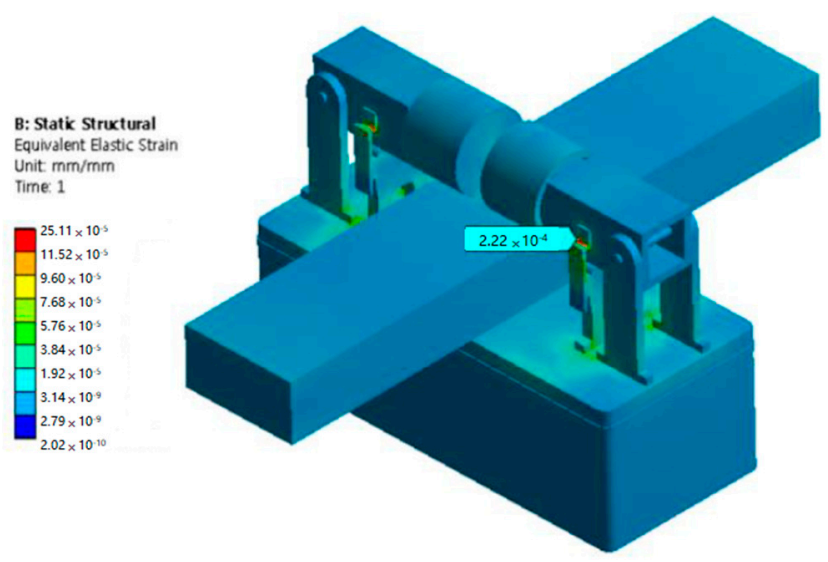

(b)

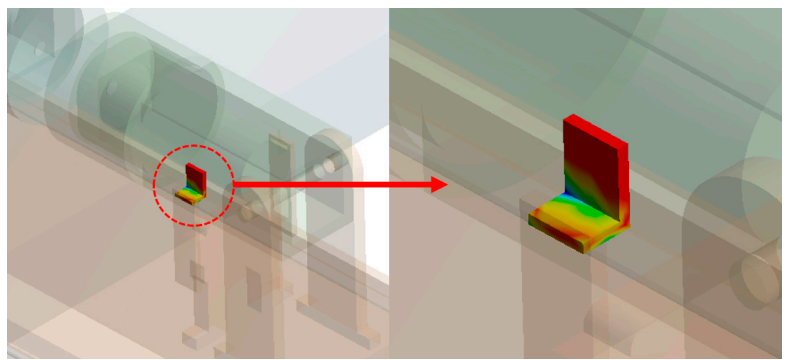

(c)

Figure 6. FEM results of the proposed overhang-type rail mover: (a) mechanical stress; (b) elastic strain; (c) safety factor.

\subsection{Position Control of Dual BLAC Motor Drives}

Simple Hall sensors are used to drive the BLAC motors which have almost sinusoidal back EMF.

For the torque and speed control, continuous rotor position has to be estimated by the Hall sensors. The rotor position is calculated by the Hall sensor position and motor speed as follows:

$$
\theta_{m(k)}=\theta_{h s(n)}+\int \omega_{m(k)} \cdot d t
$$

where $\theta_{m(k)}$ is the calculated present rotor position, and $\theta_{h s(n)}$ is the rotor position determined by the Hall sensor signal $n . \omega_{m(k)}$ is the motor speed. 
The moving length of the mover is controlled indirectly through motor speed. Hall sensor pulses are used to determine the position.

Figure 7 shows the conventional position control scheme of a dual-motor drive. In the figure, $T_{m 1}{ }^{*}$ and $T_{m 2}{ }^{*}$ are the torque references of each motor to adjust the motor speed. Motor speed reference is changed accordingly to control the motor position. In the figure, $x_{m}{ }^{*}$ denotes the reference position. $\omega_{m 1}{ }^{*}$ and $\omega_{m 2}{ }^{*}$ are speed references of each motor.

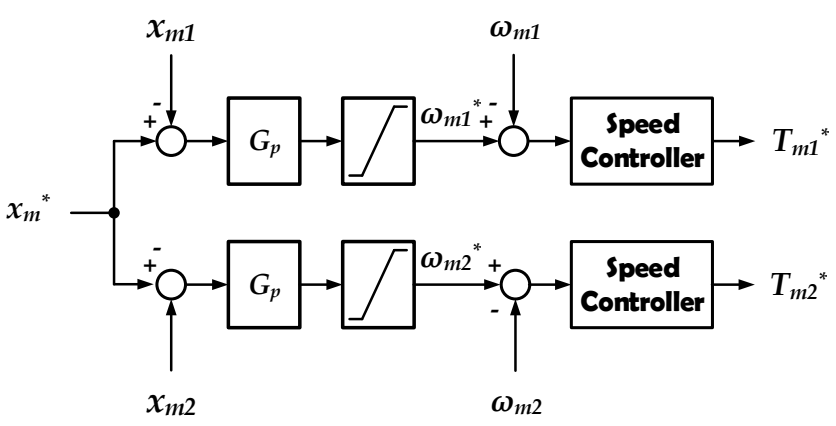

Figure 7. Moving length control scheme of dual motor drives.

The final motor speed references to satisfy the moving length can be determined as follows:

$$
\begin{aligned}
& \omega_{m 1}{ }^{*}=G_{p} \cdot\left(x_{m}{ }^{*}-x_{m 1}\right) \\
& \omega_{m 2}{ }^{*}=G_{p} \cdot\left(x_{m}{ }^{*}-x_{m 2}\right)
\end{aligned}
$$

where $G_{p}$ is the gain position controller. $x_{m 1}$ and $x_{m 2}$ are the actual moving lengths of each motor. However, as stated before, the actual translation speed and end-position can be different between the two motors, especially when only a Hall sensor-based method is used, because the resolution is low.

\section{Proposed Position Control Scheme}

Sudden acceleration generates mechanical vibration. Proper acceleration and deceleration slopes are important in reducing this effect. In the proposed position control scheme, the speed reference model using the cosine acceleration slope is adopted.

Figure 8 shows the proposed cosine acceleration slope speed reference model. The reference moving position $x_{m}{ }^{*}$ can be divided according to the three speed reference regions: acceleration, constant speed, and deceleration regions, each during $t_{a c c}, t_{c o n}$, and $t_{d e c}$, respectively. The maximum speed $\omega_{\max }{ }^{*}$ is set to satisfy the moving velocity requirements of the rail mover. As shown in Figure 8, the minimum speed reference $\omega_{\text {min }}{ }^{*}$ is set in the deceleration region to reduce the speed ripple in the low-speed region.

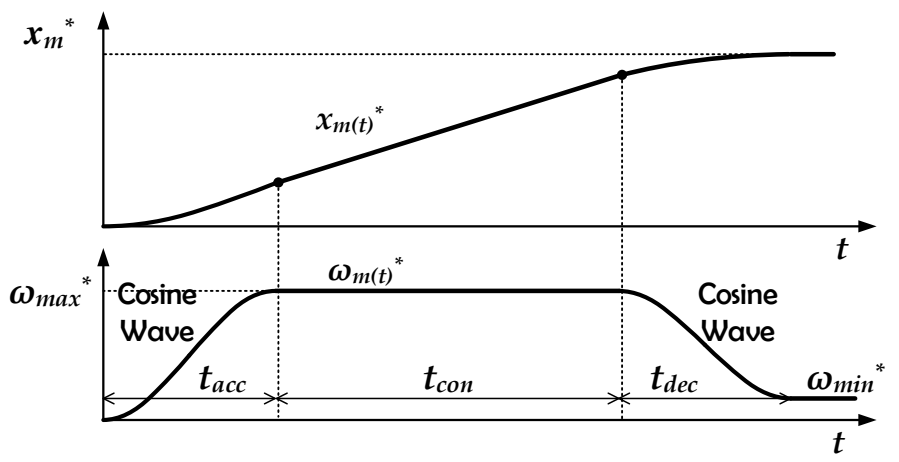

Figure 8. Proposed cosine acceleration speed reference model.

As is well known, the speed control performance based on a simple Hall sensor is greatly reduced in the low-speed region due to the sensor resolution [9]. In order to reduce 
the speed ripple by speed sensing resolution in the low-speed region, the speed reference around the stop region is restricted by the minimum controllable speed $\omega_{\text {min }}{ }^{*}$ in Figure 8 .

According to the operating time $t$, the instantaneous speed reference and moving position reference can be derived as follows:

$$
\begin{gathered}
\omega_{m(t)}{ }^{*}=\frac{\omega_{m a x}{ }^{*}}{2} \cdot\left(1-\cos \left(\frac{\pi}{t_{a c c}}\right) \cdot t\right), \text { when } t \leq t_{a c c} \\
\omega_{m(t)}{ }^{*}=\omega_{m a x}{ }^{*}, \text { when } t \leq\left(t_{a c c}+t_{c o n}\right) \\
\omega_{m(t)}{ }^{*}=\frac{\omega_{m a x}{ }^{*}}{2} \cdot\left(1+\cos \left(\frac{\pi}{t_{a c c}}\right) \cdot\left(t-t_{a c c}-t_{c o n}\right)\right), \text { when } t \geq\left(t_{a c c}+t_{c o n}\right) \\
x_{m(t)}{ }^{*}=r \cdot g_{n}-1 \int \omega_{m(t)}{ }^{*} \cdot d t
\end{gathered}
$$

where $r$ denotes the radius of roller, and $g_{n}$ is the gear ratio. According to the required moving velocity, the total run-time and the speed of the motor can be calculated using the cosine slope function for smooth acceleration. The speed references for each motor can be derived using the instantaneous speed reference based on the acceleration slope and position controller. Although mechanical vibration of the rail mover can be reduced by this method, the moving length difference between the two motors still has to be compensated for to improve the control performance.

To achieve this, a simple position difference between the two motors can be compensated through proportional compensation for each speed references as shown in Figure 9.

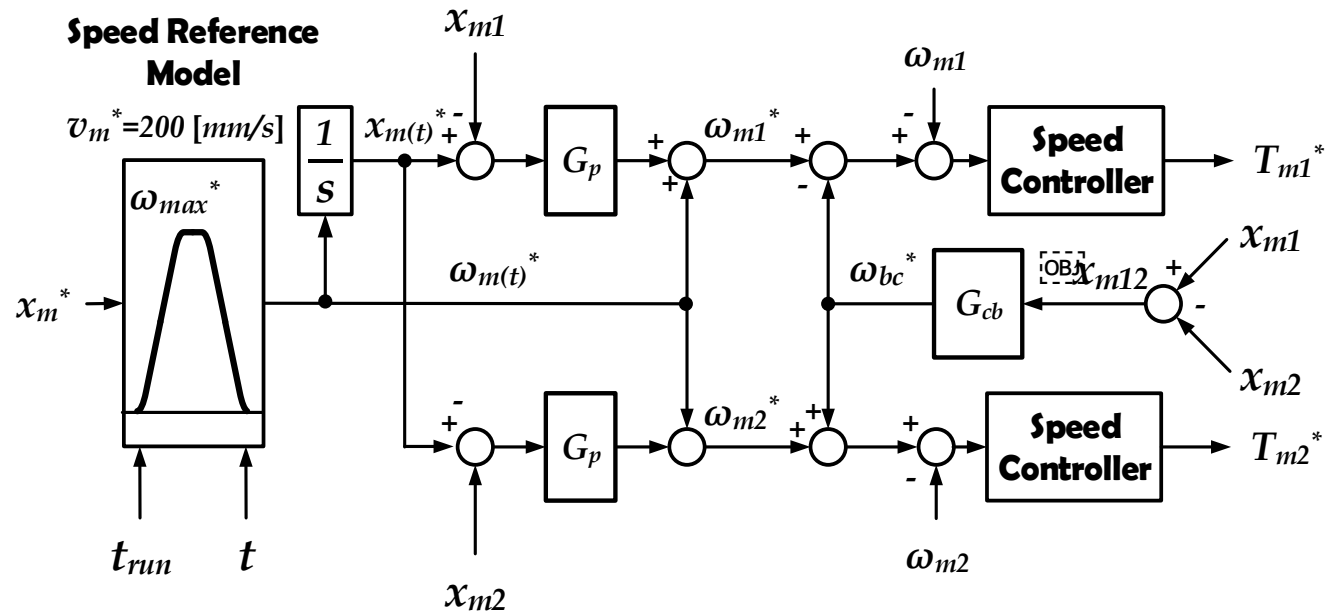

Figure 9. Simple balancing compensation control scheme.

In the proposed speed reference model, the position controller can be expressed as follows:

$$
\begin{aligned}
& \omega_{m 1}{ }^{*}=\omega_{m(t)}{ }^{*}+G_{p} \cdot\left(x_{m(t)}{ }^{*}-x_{m 1}\right) \\
& \omega_{m 2}{ }^{*}=\omega_{m(t)}{ }^{*}+G_{p} \cdot\left(x_{m(t)}{ }^{*}-x_{m 2}\right)
\end{aligned}
$$

where $\omega_{m(t)}{ }^{*}$ and $x_{m(t)}{ }^{*}$ are the instantaneous speed and position references according to operating time. The position difference of the two motors and the compensation term can be determined as follows:

$$
\begin{gathered}
\Delta x_{m 12}=x_{m 1}-x_{m 2} \\
\omega_{b c}{ }^{*}=G_{b c} \cdot \Delta x_{m 12}
\end{gathered}
$$


where $\Delta x_{m 12}$ denotes the position difference between the two motors, and $G_{b c}$ is compensator gain. The motor speed references can be obtained by the compensation as follows:

$$
\begin{aligned}
& \omega_{m 1 c}{ }^{*}=\omega_{m 1}{ }^{*}-\omega_{b c}{ }^{*} \\
& \omega_{m 2 c}{ }^{*}=\omega_{m 2}{ }^{*}+\omega_{b c}{ }^{*}
\end{aligned}
$$

Using this simple compensation, the position difference between the two motors can be reduced, and smooth movement without roller collision can be achieved. Still, the position difference cannot be fully compensated if sudden unbalanced load occurs on either side of the motor, such as if the guide roller collides. The undesired non-linear load decreases the position control performance.

The tuning of the gains is important as high compensation gain $G_{b c}$ can create an additional speed vibration. The same rule applies to the position controller gain $G_{p}$. The one-sided non-linear load creates a difference in moving speed, and the mover will move with disturbed posture. To solve this problem, this position error is controlled in the proposed control scheme. Figure 10 shows the block diagram of the proposed position control scheme for the dual BLAC motor.

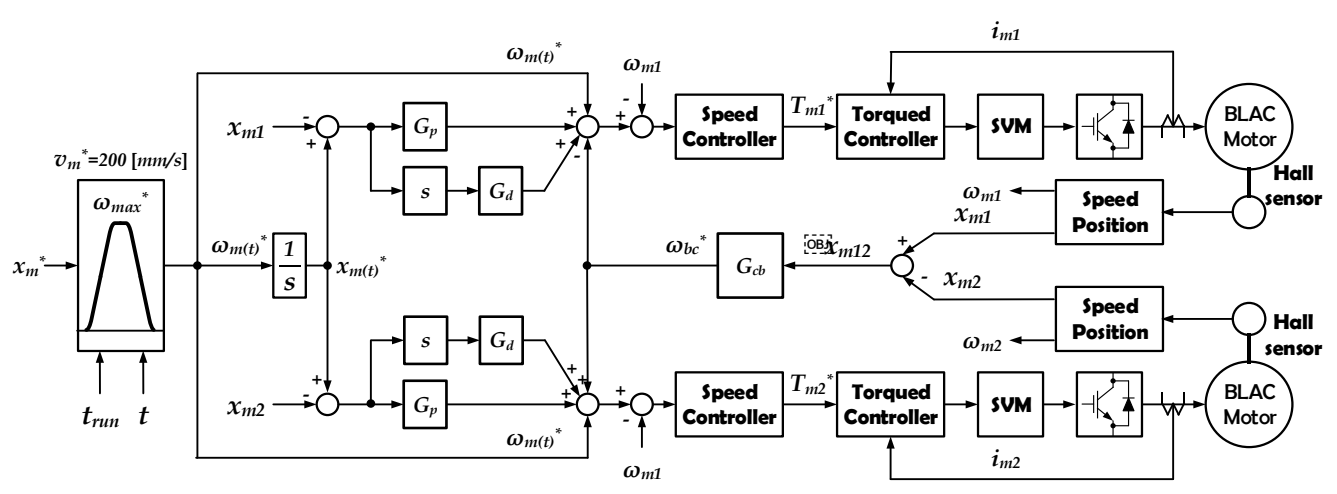

Figure 10. Block diagram of the proposed position control scheme.

For both motors, the position controller can be described as follows:

$$
\begin{gathered}
\Delta x_{m 1}=x_{m(t)}{ }^{*}-x_{m 1} \\
\Delta x_{m 2}=x_{m(t)}{ }^{*}-x_{m 2} \\
\omega_{m 1 s}{ }^{*}=\omega_{m(t)}{ }^{*}+G_{p} \Delta x_{m 1}+G_{d} \frac{\Delta x_{m 1}}{d t}-\omega_{b c}{ }^{*} \\
\omega_{m 2 s}{ }^{*}=\omega_{m(t)}{ }^{*}+G_{p} \Delta x_{m 2}+G_{d} \frac{\Delta x_{m 2}}{d t}+\omega_{b c}{ }^{*}
\end{gathered}
$$

where $G_{d}$ is the differential control gain. The additional differential control term can suppress the sudden position error variation from the one-sided non-linear load variation and balance the mover. Steady-state position error can be regulated by the designed proportional controller based on the instantaneous real-time speed and position reference model. The differential control gain can only restrict the sudden variation of motion of the designed rail mover in the balanced motion.

\section{Experimental Results}

In order to verify the proposed control scheme and the designed overhang-type rail mover system, the rail guide system was manufactured. Table 1 shows the detailed specifications of the designed rail mover. The mover is driven by two BLAC motors with simple Hall sensors attached to detect the rotor position. The ratings of the two motors are the same as presented in Table 2. The back EMF of the BLAC motor is not a perfect ideal sinusoidal waveform but is close to it. 
Table 1. Specifications of the overhang-type mover.

\begin{tabular}{cccc}
\hline Parameter & Value & Parameter & Value \\
\hline Gear Ratio & $26: 1$ & Total Length & $533.2 \mathrm{~mm}$ \\
Total Height & $510.2 \mathrm{~mm}$ & Width & $233.2 \mathrm{~mm}$ \\
Upside Height & $258.6 \mathrm{~mm}$ & Inside Width & $183.2 \mathrm{~mm}$ \\
Rail Width & $160.0 \mathrm{~mm}$ & Rail Height & $80.0 \mathrm{~mm}$ \\
\hline
\end{tabular}

Table 2. Specifications of the traction BLAC motor.

\begin{tabular}{cccc}
\hline Parameter & Value & Parameter & Value \\
\hline Power & $180 \mathrm{~W}$ & Voltage & $24 \mathrm{~V}$ \\
Rated Current & $7 \mathrm{~A}$ & Rated Speed & $4200 \mathrm{r} / \mathrm{min}$ \\
Phase Resistance & $0.0894 \Omega$ & Phase Inductance & $0.122 \mathrm{mH}$ \\
Pole-pair & 8 & Tire Radius & $115 \mathrm{~mm}$ \\
\hline
\end{tabular}

Figure 11 shows the manufactured rail mover and the dual motor drive. A geared BLAC motor is placed inside the motor housing, which is fixed using a hinge. The fixation hinge makes it easy to attach/detach the mover.

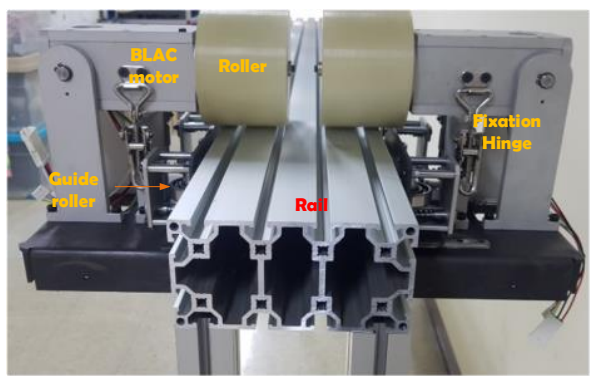

Figure 11. Manufactured rail mover.

For the dual BLAC motor control, a TMS320F28065 DSP (Digital Signal Processor) by TI (Texas Instruments) is used. The phase currents of the motor are fed to ACS725 chip-type current sensors. At the bottom, an IPM (Intelligent Power Module) is soldered to execute the DC bus voltage switching. The current controller is designed with $100 \mu \mathrm{s}$ of sampling period with $10 \mathrm{kHz}$ of switching frequency. The motor speed is calculated with Hall-sensor signal capture.

In order to verify the proposed position control scheme, the conventional position control method, balance compensating, and the proposed control scheme are compared with the same acceleration model. The target moving length is set to $1 \mathrm{~m}$, and the required moving velocity is $200 \mathrm{~mm} / \mathrm{s}$.

Figure 12 shows the experimental results at no-load condition on the rail. As shown in Figure 12a, the rail mover did follow the target position, but there was a $1.2 \mathrm{~mm}$ position difference between the two motors. In the no-load condition, a simple balance compensating method and the proposed control scheme can reduce the position difference and position error altogether. In Figure 12, $\Delta x_{m 12}$ denotes the instantaneous moving length difference between motor 1 and 2 and, it can be calculated as $\Delta x_{m 12}=x_{m 1}-x_{m 2}$. By description, $\Delta x_{m 12}$ explains the balance error of the dual traction BLAC motor in the proposed system. 


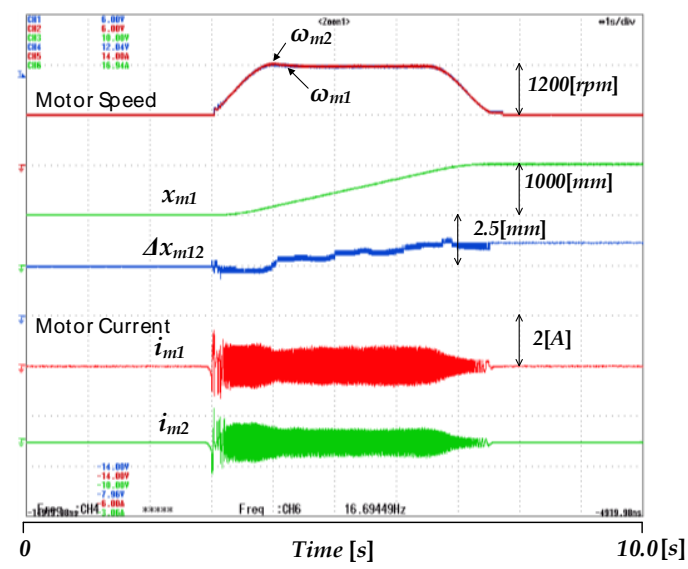

(a)

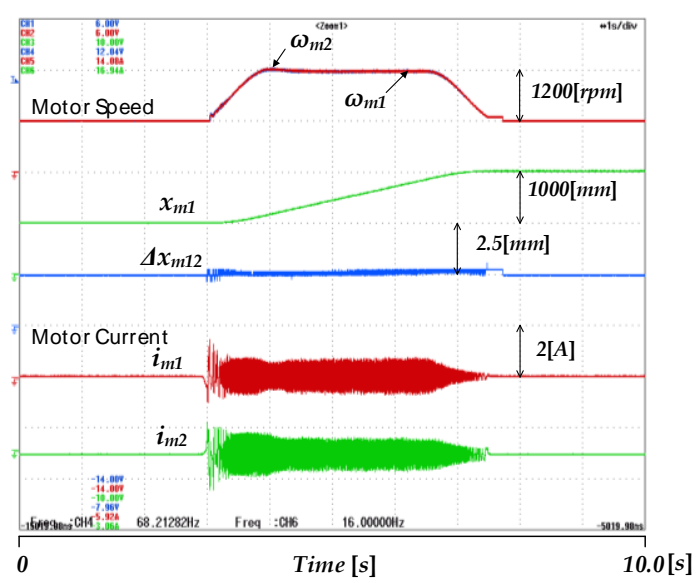

(b)

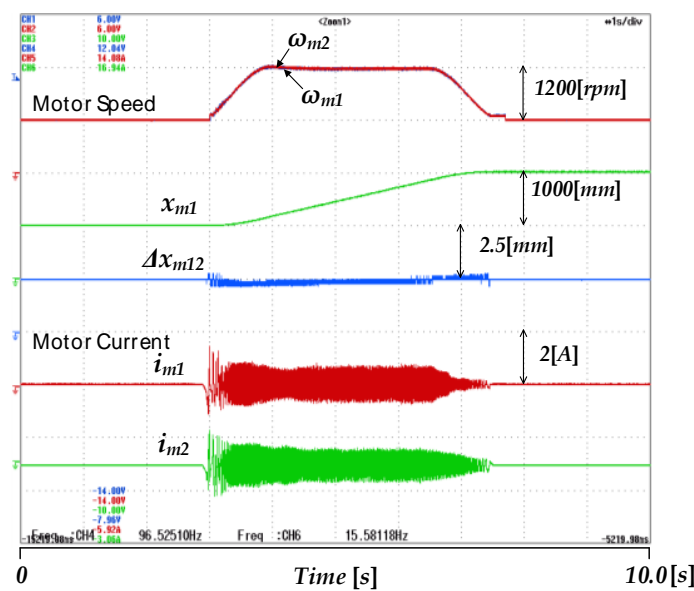

(c)

Figure 12. Experimental result in no-load condition: (a) Conventional position control method; (b) Balancing position control method; (c) Proposed control method.

Figure 13 shows the experimental results at $10 \mathrm{~N}$ continuous load injection on motor 2 for $1.5 \mathrm{~s}$. In the conventional position control method, as the load starts and compensation proceeds, the position difference does decrease, but then it rises again. With the balancing control method, the overall difference is relatively small, but it is biased towards one side. On the other hand, in the proposed control method, the position difference, which is close 
to zero, is maintained regardless of whether there is load, and the final position difference is also zero.

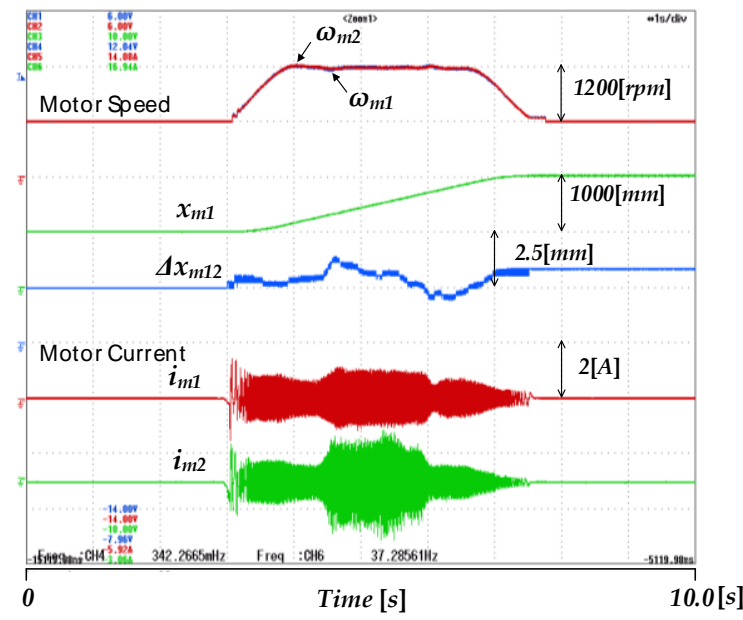

(a)

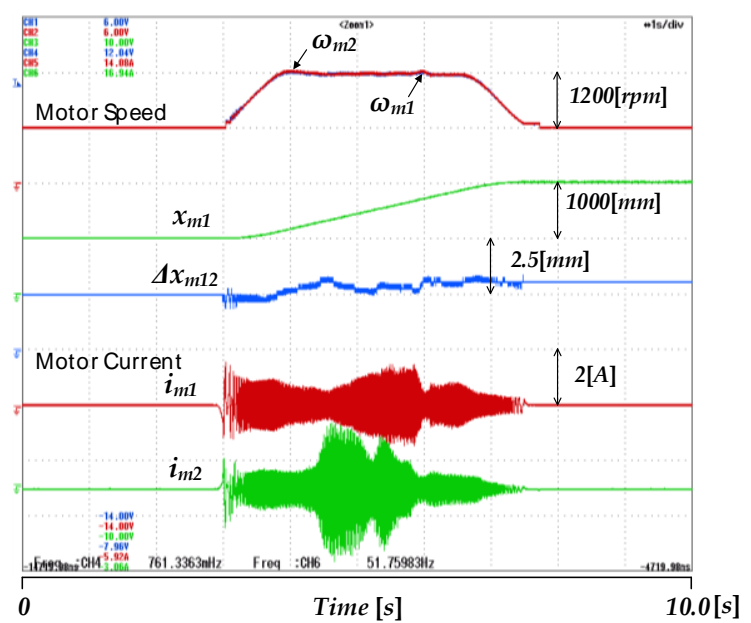

(b)

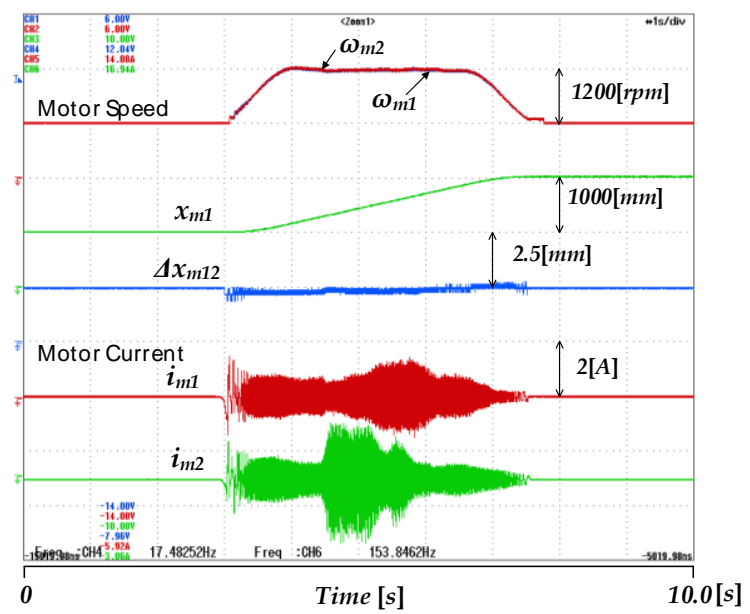

(c)

Figure 13. Experimental result of $10 \mathrm{~N}$ continuous load on the right motor: (a) conventional position control method; (b) balancing position control method; (c) proposed control method. 
In Figure 14a, it is shown that the real-time position difference, due to repetitive load, cannot be compensated for by the conventional position control method. In Figure 14b, the position difference was relatively reduced due to the balancing control between the two motors. In the Figure 14c, it is confirmed that the amplitude of the position difference was significantly reduced and the mover moves up to its final stop point with zero error.

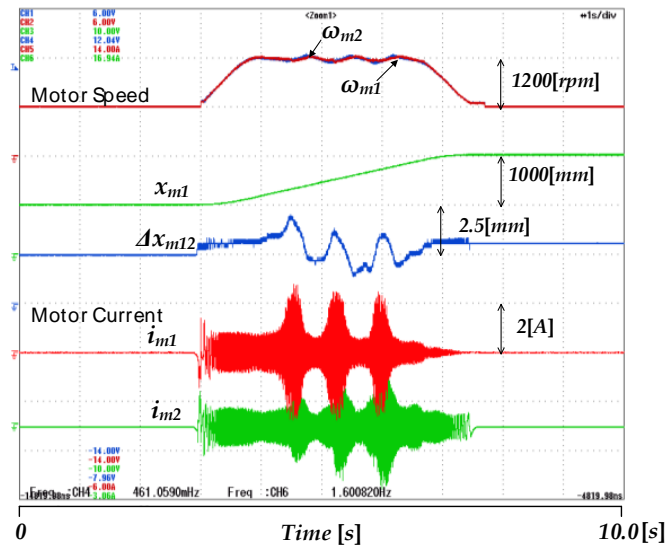

(a)

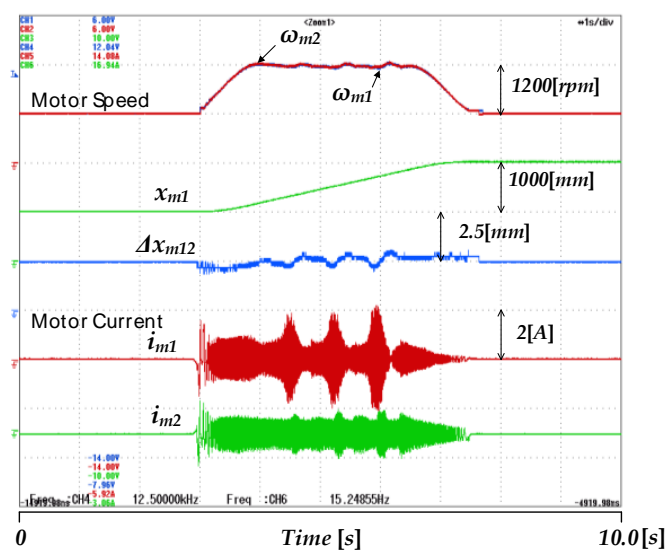

(b)

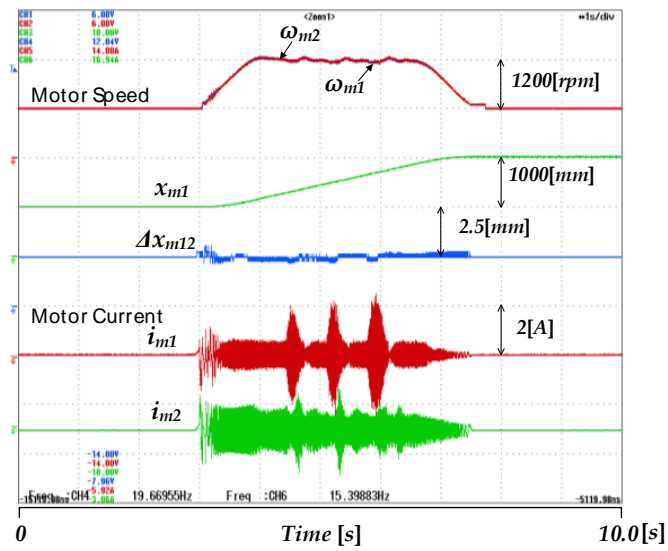

(c)

Figure 14. Experimental result of $10 \mathrm{~N}$ repetitive load on the left motor: (a) conventional position control method; (b) balancing position control method; (c) proposed control method. 
Through the comparison of the experiment results, the proposed balanced position control with a Proportional-Differential (PD) position controller and the instantaneous speed reference model shows a significant reduction in position and balance error between the two motors in various load conditions.

\section{Conclusions}

This paper presents the design and moving position control of an overhang-type rail mover using a dual BLAC motor to reduce position error and moving difference between the two motors. The overhang structure is proposed to reduce the slip effect between the traction roller and rail surface by pressing the traction mover weight on the top surface of the rail. The dual BLAC motor is used for traction on the top surface of the rail to avoid fixing the rail onto the ceiling.

The moving position of the designed rail mover is controlled by a simple Hall position sensor of the BLAC motor. Because of the resolution of the Hall sensor, the speed and position control performance of the motor in the low-speed region are not good. Furthermore, the position error and the moving length difference between the two motors can create additional unwanted friction on the rail.

In order to improve the control performance of the proposed system, a PD position control scheme with balance compensation based on the instantaneous speed and position reference model is proposed. Considering the low resolution of a Hall position sensor, minimum speed is considered in the reference model. The moving position and speed can be controlled at the same time using the simple proportional gain of the real-time position error and speed reference trajectory. Then, the additional differential gain can limit the sudden variation of the traction BLAC motor, and the balance compensation can adjust the moving direction for the motors.

Through experiments, it can be seen that the proposed position control scheme reduces the rail mover position error at the end position. Furthermore, the moving position error between two motors is greatly reduced thanks to the simple balance compensation at the sudden load variation.

Author Contributions: Conceptualization, K.C. and D.-H.L.; validation, K.C.; resources, D.-H.L.; writing, K.C.; supervision, D.-H.L. All authors have read and agreed to the published version of the manuscript.

Funding: This research was supported by Korea Electric Power Corporation (Grant number: R19XO0106) and BB21+ Project in 2020.

Institutional Review Board Statement: Not applicable.

Informed Consent Statement: Not applicable.

Data Availability Statement: Data sharing not applicable.

Conflicts of Interest: The authors declare no conflict of interest.

\section{References}

1. Koivo, A.; Ten-Huei, G. Adaptive Linear Controller for Robotic Manipulators. IEEE Trans. Autom. Control 1983, 28, 162-171. [CrossRef]

2. Xu, J.; Liu, J.; Sheng, J.; Liu, J. Arc Path Tracking Algorithm of Dual Differential Driving Automated Guided Vehicle. In Proceedings of the 2018 11th International Congress on Image and Signal Processing, BioMedical Engineering and Informatics (CISP-BMEI), Beijing, China, 13-15 October 2018; pp. 1-7.

3. Barankova, I.; Mikhailova, U.; Lukianov, G. Automated Control System of a Factory Railway Transport Based on ZigBee. In Proceedings of the 2016 2nd International Conference on Industrial Engineering, Applications and Manufacturing (ICIEAM), Chelyabinsk, Russia, 19-20 May 2016; pp. 1-5.

4. Hossain, S.G.M.; Jamil, H.; Ali, M.Y.; Haq, M.Z. Automated Guided Vehicles for Industrial Logistics—Development of Intelligent Prototypes Using Appropriate Technology. In Proceedings of the 2010 The 2nd International Conference on Computer and Automation Engineering (ICCAE), Singapore, 26-28 February 2010; pp. 237-241.

5. Yamamoto, S. Development of Inspection Robot for Nuclear Power Plant. In Proceedings of the 1992 IEEE International Conference on Robotics and Automation, Nice, France, 14 May 1992; pp. 1559-1566. 
6. Kim, D.; Lee, S.; Kang, M.; Chun, B.; Han, C. Proposal of Built-in-Guide-Rail Type Building Façade Cleaning Robot and Its Motion Planning Algorithm. In Proceedings of the 2012 IEEE International Conference on Automation Science and Engineering (CASE), Seoul, Korea, 20-24 August 2012; pp. 1004-1009.

7. Wang, L.; Liu, F.; Xu, S.; Cheng, S.; Zhang, J. Design and Analysis of a Line-Walking Robot for Power Transmission Line Inspection. In Proceedings of the 2010 IEEE International Conference on Information and Automation, Harbin, China, 20-23 June 2010; pp. 1398-1403.

8. Wang, L.; Liu, F.; Wang, Z.; Xu, S.; Cheng, S.; Zhang, J. Development of a Novel Power Transmission Line Inspection Robot. In Proceedings of the 2010 1st International Conference on Applied Robotics for the Power Industry, Montreal, QC, Canada, 5-7 October 2010; pp. 1-6.

9. Bae, J.; Lee, D. Position Control of a Rail Guided Mover Using a Low-Cost BLDC Motor. IEEE Trans. Ind. Appl. 2018, 54, 2392-2399. [CrossRef]

10. Zhakov, A.; Zhu, H.; Siegel, A.; Rank, S.; Schmidt, T.; Fienhold, L.; Hummel, S. Automatic Fault Detection in Rails of Overhead Transport Systems for Semiconductor Fabs. In Proceedings of the 2019 30th Annual SEMI Advanced Semiconductor Manufacturing Conference (ASMC), Saratoga Springs, NY, USA, 6-9 May 2019; pp. 1-6.

11. Borgerink, D.J.; Stegenga, J.; Brouwer, D.M.; Wörtche, H.J.; Stramigioli, S. Rail-Guided Robotic End-Effector Position Error Due to Rail Compliance and Ship Motion. In Proceedings of the 2014 IEEE/RSJ International Conference on Intelligent Robots and Systems, Chicago, IL, USA, 14-18 September 2014; pp. 3463-3468.

12. Viegas, C.; Tavakoli, M.; Lopes, P.; Dessi, R.; de Almeida, A.T. SCALA-A Scalable Rail-Based Multirobot System for Large Space Automation: Design and Development. IEEE/ASME Trans. Mechatron. 2017, 22, 2208-2217. [CrossRef]

13. Cupertino, F.; Naso, D.; Mininno, E.; Turchiano, B. Sliding Mode Control with Double Boundary Layer for Robust Compensation of Payload Mass and Friction in Linear Motors. In Proceedings of the 2008 IEEE Industry Applications Society Annual Meeting, Portland, OR, USA, 5-9 October 2008; pp. 1-8.

14. Liu, X.; Cao, H.; Wei, W.; Wu, J.; Li, B.; Huang, Y. A Practical Precision Control Method Base on Linear Extended State Observer and Friction Feedforward of Permanent Magnet Linear Synchronous Motor. IEEE Access 2020, 8, 68226-68238. [CrossRef]

15. Zhao, J.; Jiang, X.; Cheng, W.; Zhao, J.; Wang, H.; Gong, K. Precise Position Detection of Linear Motor Movers Based on Extended Joint Transformation Correlation. IEEE Trans. Ind. Inform. 2020, 16, 814-822. [CrossRef]

16. Chen, D.; Yin, J.; Chen, L.; Xu, H. Parallel Control and Management for High-Speed Maglev Systems. IEEE Trans. Intell. Transp. Syst. 2017, 18, 431-440. [CrossRef]

17. Zhou, H.; Deng, H.; Duan, J. Hybrid Fuzzy Decoupling Control for a Precision Maglev Motion System. IEEE/ASME Trans. Mechatron. 2018, 23, 389-401. [CrossRef]

18. Smoczek, J.; Szpytko, J. Particle Swarm Optimization-Based Multivariable Generalized Predictive Control for an Overhead Crane. IEEE/ASME Trans. Mechatron. 2017, 22, 258-268. [CrossRef]

19. Sun, N.; Yang, T.; Fang, Y.; Wu, Y.; Chen, H. Transportation Control of Double-Pendulum Cranes with a Nonlinear Quasi-PID Scheme: Design and Experiments. IEEE Trans. Syst. ManCybern. Syst. 2019, 49, 1408-1418. [CrossRef]

20. Xing, X.; Liu, J. PDE Modelling and Vibration Control of Overhead Crane Bridge with Unknown Control Directions and Parametric Uncertainties. IET Control Theory Appl. 2019, 14, 116-126. [CrossRef]

21. Tomizuka, M.; Hu, J.-S.; Chiu, G.; Kamano, T. Synchronization of Two Motion Control Axes Under Adaptive Feedforward Control. J. Dyn. Syst. Meas. Control Trans. 1992, 114, 196-203. [CrossRef]

22. Alain, B.; Maria, P.-D.; Phillipe, D.; Rosendo, P.-E.; Paul-Etienne, V.; Xavier, K. Weighted Control of Traction Drives with Parallel-Connected AC Machines. IEEE Trans. Ind. Electron. 2006, 53, 1799-1806.

23. Sven, G.R.; Lorenz, H.; Sameer, K.; Klas, N. Precise Robot Motions using Dual Motor Control. In Proceedings of the 2010 IEEE International Conference on Robotics and Automation, Anchorage, AK, USA, 3-8 May 2010; pp. 5613-5620.

24. Xiao, Y.; Zhu, K.Y. Optimal Synchronization Control of High-precision Motion System. IEEE Trans. Ind. Electron. 2006, 53, 1160-1169. [CrossRef]

25. Wei, C.; Yifei, W.; Renhui, D.; Qingwei, C.; Xiaobei, W. Speed Tracking and Synchronization of a Dual-Motor System via Second Order Sliding Mode Control. Math. Probl. Eng. 2013, 2013, 1-10.

26. Mikho, M. Control of Dual-Motor Drive Systems. In Proceedings of the International Conference on Mechatronics and Information Technology 2001, Como, Italy, 8-12 July 2001; pp. 1-6. 